\title{
Cancer-Associated Lymphatic and Venous Vessels in Colonic Carcinomas
}

\author{
Tatsuo Tomita ${ }^{1,2,3}$ \\ ${ }^{1}$ Department of Integrative Bioscience, Oregon Health and Science University, Portland, Oregon, USA \\ ${ }^{2}$ Department of Pathology, Oregon Health and Science University, Portland, Oregon, USA \\ ${ }^{3}$ Oregon National Primate Center, Oregon Health and Science University, Portland, Oregon, USA \\ Email: tomitat@ohsu.edu
}

Received 9 January 2014; revised 9 February 2014; accepted 9 March 2014

Copyright (C) 2014 by author and Scientific Research Publishing Inc.

This work is licensed under the Creative Commons Attribution International License (CC BY).

http://creativecommons.org/licenses/by/4.0/

(c) (i) Open Access

\section{Abstract}

Objective: Colonic carcinomas spread to regional lymph nodes and liver. There are cancer-associated lymphatic and venous vessels at the margin of colonic carcinomas, which facilitate spreading carcinoma through lymphatic and venous vessels. This study aimed to examine cancer-associated lymphatic and venous vessels in $\mathrm{TNM}_{\mathrm{T}_{1}}$ to $\mathrm{T}_{3}$ carcinomas using lymphatic vessel hyaluronan receptor for lymphatic vessels and von Willebrand factor for venous vessels by immunocytochemical staining. Materials and Methods: $A$ total of 40 cases of moderately differentiated colonic carcinoma were studied using routinely formalin-fixed and paraffin-embedded sections. The cases consisted of 10 cases of $T N M T_{1}, 15$ cases each of $T_{2}$ and $T_{3}$ cases. Immunocytochemical staining was performed using goat antihuman LYVE-1for lymphatic vessels and rabbit antihuman von Willebrand factor for venous vessels. Results: In TNM T ${ }_{1}$ carcinoma, increased, irregular and narrow lymphatic and venous vessels were present in the adjacent normal mucosa to the carcinoma, some of which penetrated cancerous lesion. There were no tumor emboli in lymphatic and venous vessels. In TNM $\mathrm{T}_{2}$ carcinoma, there were few lymphatic and venous vessels in midst of the carcinoma whereas numerous small lymphatic and venous vessels were present within muscle layers adjacent to the invading carcinoma. Extramural tumor embolus was present in submucosa in one case. In TNM $T_{3}$ carcinoma, cancer has invaded through the muscle layers where dilated lymphatic and venous vessels were present adjacent to cancerous nests. Tumor emboli were identified in two cases by immunocytochemical staining. Conclusion: The current study showed cancer-associated lymphatic and venous vessels at the interface in TNM $\mathrm{T}_{1}$ carcinoma to dilated intramuscular lymphatic and venous vessels adjacent to invading cancerous nests in TNM $T_{3}$ carcinoma, and supports cancerous cells spread via lymphatic and venous vessels through muscle layers to subserosa as supported by tumor emboli in the lymphovascular system. 
Keywords

Colonic Carcinoma, Factor-8, Lymphatic Vessels, LYVE-1, Venous Vessels

\section{Introduction}

The most common sites of metastasis of colonic carcinoma are regional lymph nodes and liver [1]. Lymph node involvement and number of metastatic lymph nodes are considered to be an important clinicopathological factor in colorectal carcinoma [1]-[5]. Since cancer cells metastasize to regional lymph nodes through lymphatic vessels and to liver through venous vessels, lymphatic and venous vessel tumor invasion is the histopathological risk factor for colorectal carcinoma [6]. It is not easy to definitely identify and evaluate lymphatic vessel invasion by routine $\mathrm{H}$. and $\mathrm{E}$. sections, and immunocytochemical identification for the lymphatic vessel is a currently available tool for identifying lymphatic vessels [7]. There is an agreement on the evidence that more lymphatic and venous vessels are present at the margins of colonic carcinoma, suggesting that colonic carcinoma spreads to regional lymph nodes through lymphatic vessels [1]-[5] and to liver by hematogenous spread [8] [9]. But cancer-associated lymphangiogenesis has been a controversial issue [3]-[5] partly due to a lack of specific and reliable immunocytochemical markers for lymphatic endothelium [10]-[12] and a lack of universally accepted methodology to identify and evaluate lymphatic vessels in surgically resected colonic cancer specimens.

By immunocytochemical staining using lymphatic vessel endothelial hyluronan receptor-1 (LYVE-1) for lymphatic vessels and von Willebrand factor (factor-8, F-8) for venous vessels, lymphatic and venous vessels were concomitantly studied in surgically resected colonic cancer specimens since this immunocytochemical staining was previously used with colonic specimens harboring polyps and adenomas [13]. Recently, immunocytochemical staining for lymphatic vessels has been performed using Prox1, podoplanin, D2-40 and LYVE-1, the latter has been widely used as a reliable marker in the previous studies [7] [10]-[13].

\section{Materials and Methods}

All cases of moderately differentiated colonic carcinoma including the adjacent normal colon were from the University of Kansas Medical Center, collected between 1999 and 2000, and consisted of 10 cases TNM T 1,15 cases each of $T_{2}$ and $T_{3}$ stages of carcinomas with a total of 40 cases [14]. Two tissue blocks including the interface between the cancerous lesion and normal mucosa were studied for $\mathrm{T}_{1}$ cases and two sections of the interface containing the entire colonic wall thickness were studied for $T_{2}$ and $T_{3}$ cases. The surgically removed specimens were routinely fixed in buffered formalin and were embedded in paraffin. Deparaffinized sections were treated with antigen retrieval procedure using citrate buffer $\mathrm{pH}$ 6.2. Immunocytochemical staining was performed for LYVE-1 using goat antihuman LYVE-1 (R \& D System, Minneapolis, MN) at 1:100 dilution and F-8 immunocytochemical staining was performed using rabbit human F-8 (Dako System, Carpenteria, CA) at 1:100 dilution as reported before [13]. At least two sections from each tissue block at the two different levels were immunostained for LYVE-1 and F-8. Only the sections, which were adequately immunostained for both LYVE-1 and F-8, were included in this study.

\section{Results}

Normal colon: Abundant LYVE-1 positive lymphatic vessels were slender, irregularly shaped channels in submucosa including muscularis mucosa, which abruptly changed the directions and consistently extended into the base and deeper end of colonic crypt while extending into submucoa vertically through the horizontal muscularis mucosa and also numerously into lymphoid follicles, when the latter were present (Figure 1(A)). Less abundant F-8 positive venous vessels appeared of more roundish shape with plump lumens, and extended sparingly into both submucosa and the entire thickness of lamina propria onto the mucosal surface and into lymphoid follicle (Figure 1(B)). Both lymphatic and venous vessels penetrated vertically through the inner muscular layer whereas the two vessels spread horizontally in the outer longitudinal muscle layer in the tangentially cut sections against the mucosal folds (Figure 1(C) and Figure 1(D)). Both intramuscular lymphatic and venous vessels were smaller and slender than those in the submucosa (Figures 1(A)-(D)). In the parallel cut sections along the 


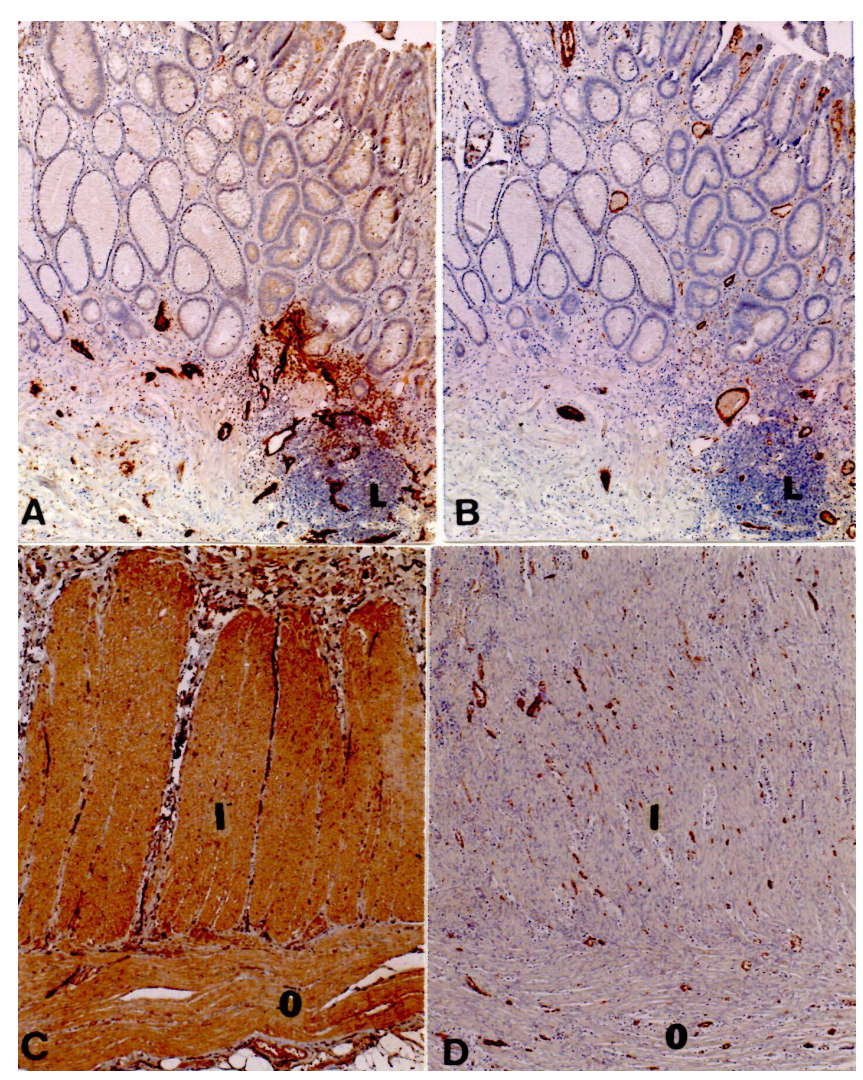

Figure 1. Normal colon. Lymphatic vessels were abundant in submucosa, penetrating through muscularis mucosa and into the base and deep-end of colonic crypt and numerously into submucosal lymphoid follicle (A). Venous vessels were relatively less numerous than lymphatic vessels in submucosa and were round with plump lumens, penetrating through muscularis mucosa sparingly into the full thickness of lamina propria onto the mucosal surface (B). Numerous small lymphatic and venous vessels penetrated vertically through the inner circular muscle layer whereas the both vessels spread horizontally in the outer longitudinal muscle layer in the tangentially cut sections against the mucosal folds (C) and (D). I: Inner muscle layer, O: Outer muscle layer, L: Lymphoid follicle. (A) and (C): LYVE-1; (B) and (D): F-8 immunostaining.

mucosal folds, both small lymphatic and venous vessels spread horizontally in the inner muscle layer, and penetrated vertically in the outer muscle layer. Thus, both lymphatic and venous vessels spread vertically and horizontally in the inner circular and outer longitudinal muscle layers, respectively (Figures 1(A)-(D)). Both intramuscular lymphatic and venous vessels were relatively smaller and slender than much more abundant irregularly shaped lymphatic vessels and plump venous vessels in the submucosa (Figures 1(A)-(D)).

Colonic carcinoma, TNM $\mathrm{T}_{1}$ : There was thin fibrous stroma between back-to-back cancerous glands, between which were a few lymphatic vessels in contrast to small but more abundant venous vessels (Figure 2(A) and Figure 2(B)). In the interface between the cancerous and normal mucosa, there were more lymphatic vessels and much more venous vessels in the adjacent normal mucosa, some of which were continuous to the cancerous mucosa (Figure 2(A) and Figure 2(B)). At the surface of cancerous mucosa, there were focally abundant small lymphatic and venous vessels in fine fibrous stroma (Figure 2(C) and Figure 2(D)). Some of the vessels in the interface were immunostained concomitantly for both LYVE-1 and F-8 (Figure 2(A) and Figure 2(B)). Mid cancerous mucosa revealed less lymphatic and venous vessels than the distant normal mucosa. The submucosa adjacent to the cancerous lesion revealed numerous slender lymphatic vessels and larger round venous vessels, 


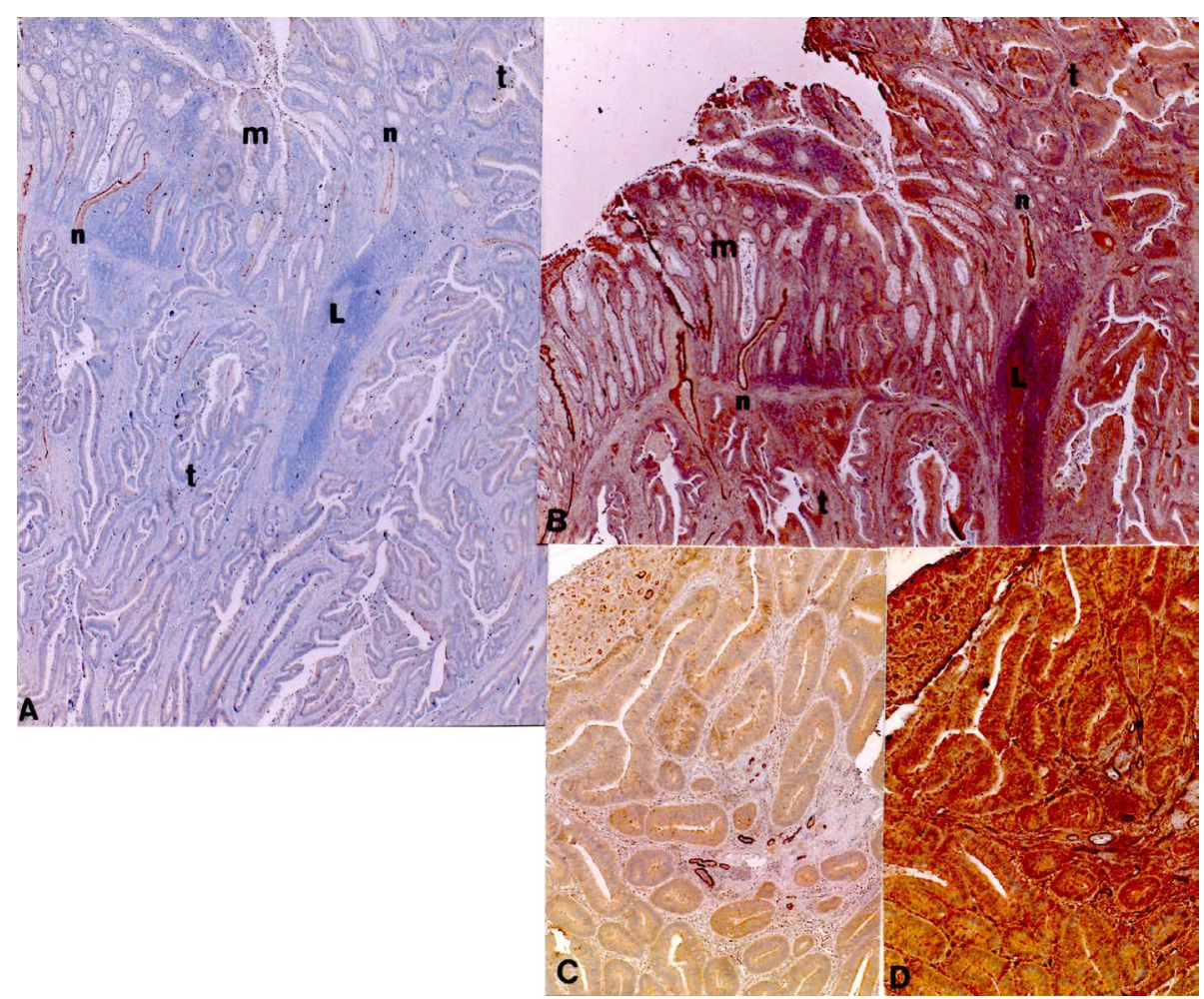

Figure 2. Interface of TNM $\mathrm{T}_{1}$ adenocarcinoma. In mucosal interface between carcinoma and normal mucosa, there were moderately increased lymphatic vessels and much more increased venous vessels in the adjacent normal mucosa than in the distant normal mucosa (A) and (B). Some small vessels (n) were positively immunostained for both LYVE-1 and F-8 (n). At the cancerous mucosal surface, there were focally increased small lymphatic vessels and more increased venous vessels in the fine fibrous stroma (C) and (D). L: Lymphoid follicle, $n$ : vessels stained for both LYVE-1 and F-8. (A) LYVE-1, (B) F-8 immunostaining.

and there were no tumor emboli in lymphatic and venous vessels.

Colonic carcinoma, $\mathrm{TNM} \mathrm{T}_{2}$ : In submucosa, there were abundant lymphatic and venous vessels with tumor emboli identified in 1 of each 15 cases of lymphatic (Figure 3(A)) and venous vessels (Figure 3(D)). Adjacent to the invading tumor within the muscle layers, there were numerous small lymphatic and larger venous vessels, which have vertically penetrated through the muscle layer (Figure 3(B) and Figure 3(C)). The majority of cancerous nests in the submucosa were surrounded by an empty halo, not representing lymphatic or venous vessels but appeared to be a contraction artifact by formalin fixation and paraffin embedding, supported by the negative immunostaining for both LYVE-1 and F-8 (Figure 3(A) and Figure 3(D)).

Clonic carcinoma, $\mathrm{TNM} \mathrm{T}_{3}$ : In muscle layers adjacent to the invading carcinoma, there were abundant small lymphatic and more abundant small venous vessels (Figure 4(A) and Figure 4(B)). Cancerous nests in the muscular layers were surrounded by small lymphatic vessels and abundant small venous vessels, some of the latter penetrated into the cancerous nests (Figure 4(C) and Figure 4(D)). In two cases, there were extramural tumor emboli in lymphatic and venous vessels out of 15 cases. In the subserosa, there were increased small lymphatic vessels and numerous, round venous vessels.

\section{Discussion}

Colonic cancer spreads predominantly to regional lymph nodes through lymphatic vessels although little is known about cancer-associated lymphangiogenesis [15] [16]. Much of the difficulty in analyzing this lymphangiogenesis is due to a lack of specific lymphatic vessel markers to differentially identify lymphatic and venous vessels by immunocytochemical staining [15]-[17]. Several lymphatic endothelial markers are now available, including LYVE-1, Prox1, podoplanin and D2-40, in which CD 34 is expressed by LYVE-1 ${ }^{+} /$podoplanin $^{+}$/ 


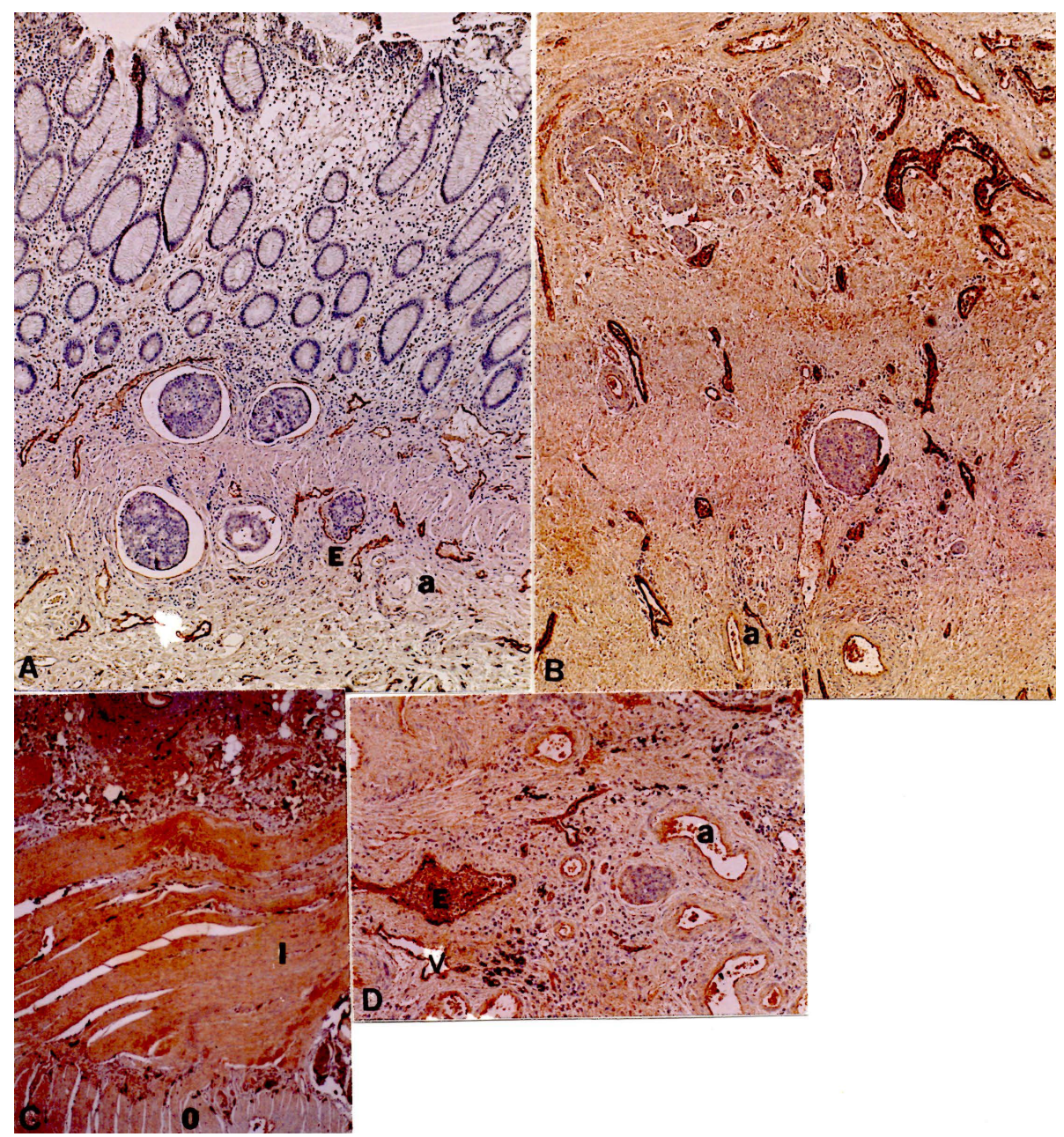

Figure 3. Submucosa and muscle layers of TNM $\mathrm{T}_{2}$ adenocarcinoma. In the submucosa adjacent to the invading cancerous nests, there were abundant slender lymphatic vessels (A) and larger and plump venous vessels (B). There were tumor emboli in submucosal lymphatic vessels (A) and venous vessels (D) whereas the majority of cancer nests in submucosa were surrounded by empty halos, not representing lymphatic or venous vessels but appeared to be a contraction artifact by formalin-fixation and paraffin-embedding (A), (C) and (D). (C) was from the section cut along the mucosal folds, showing horizontal inner muscle layer. a: artery, E: tumor embolus, I: Inner muscle layer, O: Outer muscle layer. (A) and (C) LYVE-1, (B) and (D) F-8 immunostaining.

Prox $1^{+}$cancer-associated lymphatic cells in colon, breast, lung and skin tumors whereas lymphatic endothelial cell in all normal organs does not express CD 34 by immmunofluorescense study on unfixed frozen sections [17].

Matsumoto et al. [18], Kaneko et al. [19] and Liang et al. [20] used podoplanin for "hot spots" and Zimmerman et al. [15], Gao et al. [21] and Longatto-Fielo et al. [22] used monoclonal D2-40 for lymphatic vessel microanalysis in peritumoral tissue of colonic carcinoma and came to the same conclusion that there was a correlation between high lymphatic vessel microdensity and positive lymphatic vessel invasion/lymph node metastasis although these authors did not mention the correlation between lymphatic vessel microdensity and lymphovascular tumor invasion [18]-[22]. Using LYVE-1 immunostaining, Minsky et al. initially studied 462 cases of colonic carcinoma and found 61 cases (13\%) showing lymphovascular invasion and had shown that lymphovascular invasion is an independent prognostic factor [23].

Prognosis of colonic carcinoma depends on vertical invasion of cancer cells according to TNM stages [14], and vertical lymphangiogenesis though the muscle layers to the deeper margins may facilitate lymphatic spread 


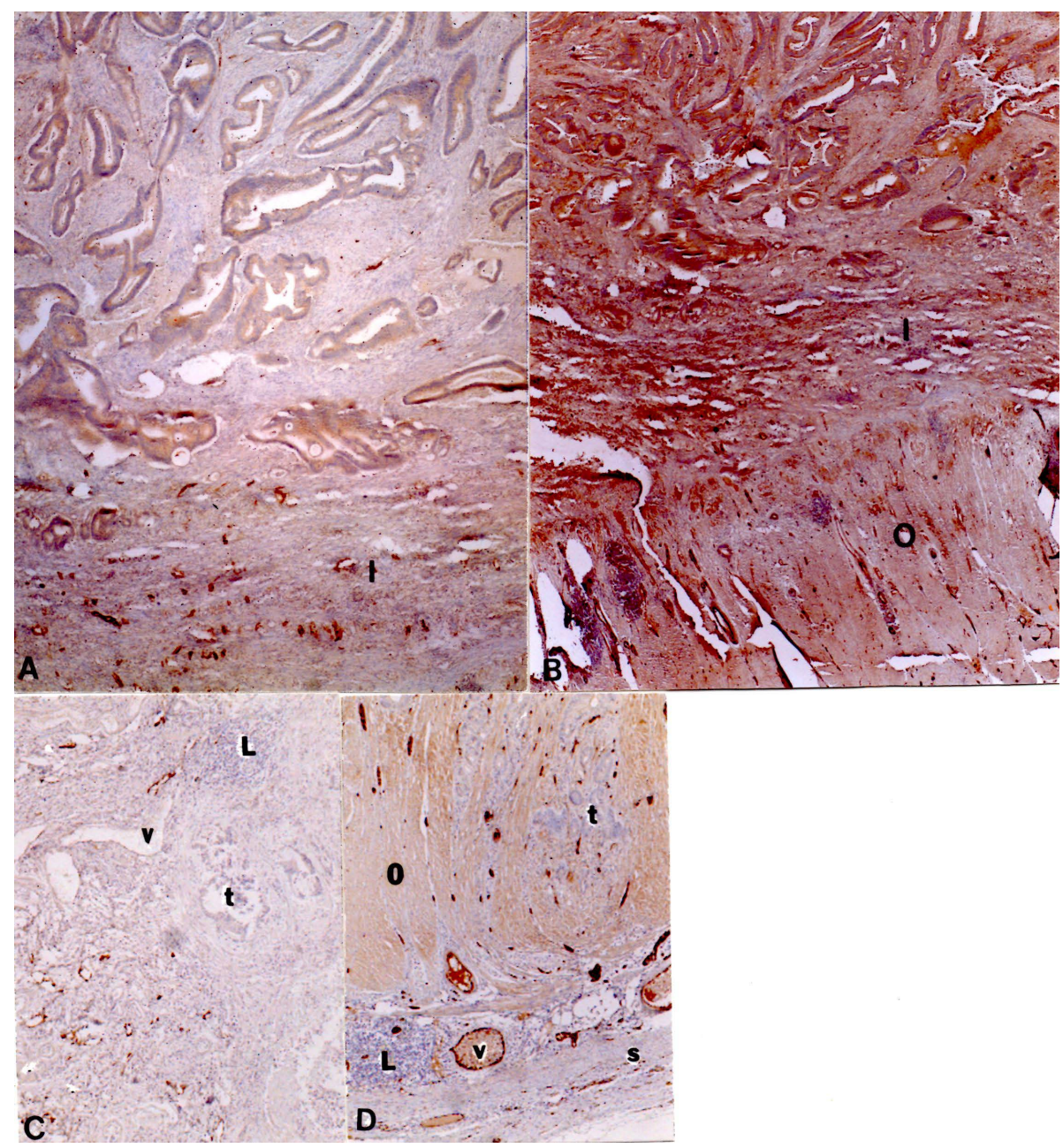

Figure 4. Deep muscle layers and subserosa of TNM $\mathrm{T}_{3}$ adenocarcinoma. There were massive cancerous masses in the muscle layers, which contained few lymphatic and venous vessels whereas there were numerous small lymphatic and venous vessels at the margins of the invading cancer (A) and (B). Adjacent to the invading cancerous nests through muscular layers, small lymphatic and round, numerous venous vessels were noted in the muscular layers (C) and (D), some of which were continuous into the cancerous nests (D). There were more round venous vessels surrounded the cancerous nests than smaller lymphatic vessels in the subserosa (C) and (D). (A)-(D) were from the sections cut along the mucosal folds, showing horizontal inner muscle layer and vertical outer muscle layer. a: artery, I: Inner muscle layer, L: Lymphoid follicle, O: Outer muscle layer, s: subserosa, t: tumor nests, v: vein. (A) and (C) LYVE-1, (B) and (D) F-8 immunostaining.

of the carcinoma. Both lymphatic and venous vessels penetrate vertically and horizontally through thicker circular inner muscle bundles and through the thinner outer longitudinal muscle layer (Figure 1 and Figure 2). Both lymphatic and venous vessels in the muscle layers are generally smaller than those in submucosa and subserosa.

The current study showed both horizontal and vertical lymphatic and venous vessel proliferation at the adjacent margins of the mucosal interface and submucosa (Figure 3(A) and Figure 3(D)) and the normal submucosa adjacent to the cancer contained abundant larger lymphatic and venous vessels. Some of the extramural vessels even contained tumor emboli as a proof for cancerous cells transported through the lymphovascular system (Figure 3(A) and Figure 3(D)). This lymphangiogenesis appeared to be cancer-associated as abundant lymphatic vessels were noted adjacent to the tumors and the invading cancerous nests (Figure 3, Figure 4). In TIM $\mathrm{T}_{3}$ carcinomas, increased, small intramuscular lymphatic vessels were present (Figure 4(C) and Figure 4(D)). 
These numerous lymphatic and venous vessels were noted adjacent to the invading carcinoma in TNM $\mathrm{T}_{2}$ and $\mathrm{T}_{3}$ cases (Figure 3 and Figure 4) in contrast to scanty lymphatic and venous vessels in mid portion of TNM $\mathrm{T}_{1}$ carcinomas (Figure 2). Therefore, cancer-associated lymphangiogenesis and angiogenesis were predominantly observed adjacent to the invading $\mathrm{T}_{2}$ and $\mathrm{T}_{3}$ carcinoma (Figure 3 and Figure 4).

Some cancer-associated small vessels were positive for both LYVE-1 and F-8 (Figure 2(A) and Figure 2(B)), suggesting newly formed cancer-associated lymphoangiogenesis from common stem cells for lymphatic and venous vessels [10]-[13]. We had also used D2-40 (Dako System) for immunostaining lymphatic vessels for lymphatic vessels, which revealed no lymphatic vessels in the mid cancerous tissue similar to those observed by LYVE-1 immunostaining (Unpublished data).

Using routinely processed formalin-fixed and paraffin-embedded sections, not all lymphatic vessels were consistently immunostained for lymphatic and venous vessels in our experience as also reported by others [13] [24] [25], most likely due to non-optimal fixation and/or poor tissue preservation for the epitopes. Furthermore, even in the well formalin-fixed and paraffin-embedded sections, lymphatic vessels were not evenly immunostained but were occasionally focally and sporadically immunostained within the same cases or even in the same microscopic slides, likely due to uneven preservation for the epitopes. It is apparent that formalin-fixation and paraffin-embedding masked or destroyed some parts of the LYVE-1 and F-8 epitopes. Fiedler et al. used frozen sections for immunofluorescence staining for multiple lymphatic endothelial markers [17] but this procedure is cumbersome and too labor intensive for a routine pathology laboratory. Complex histopathological and functional interactions between cancerous cells and lymphangiogenesis in the cancer may definitely play a pivotal role in the commonly metastasizing tumor like colonic carcinoma [26].

This current study has shown that there were abundant small extramural lymphatic and more abundant venous vessels in $\mathrm{T}_{1}$ carcinomas and, there were more abundant intramural lymphatic and venous vessels once cancer has invaded into muscle layers in TNM $\mathrm{T}_{2}$ carcinoma, and further more abundant intramural lymphatic and venous vessels appeared when the cancer invaded through the muscle layers to subserosa in TNM $\mathrm{T}_{3}$ cases. These cancer-associated lymphatic and venous vessels appeared concomitantly and may provide an easy access for cancer cells to regional lymph nodes and beyond, including hematogenous spread to liver. Lymphovascular invasion identified by tumor emboli was $0 \%$ to $15 \%$ in our cases. Harris et al. assembled a group of six GI pathologists to test an interobserver variability by examining 50 cases of moderately-differentiated $T_{1}$ to $T_{3}$ colorectal carcinoma and the tally was: $7 \%, 24 \%$ and $31 \%$ for lymphatic invasion and $0 \%, 10 \%$ and $23 \%$ for venous vessels for $T_{1}, T_{2}$ and $T_{3}$ the carcinomas, respectively [27]. There was substantial interobserver variability in diagnosing lymphovascular invasion among the six pathologists due to the different individual criteria for lymphovascualr invasion [27]. Immunocytochecmical staining for D2-40 for lymphatic vessels and CD 31 for venous vessels did not improve for identifying lymphovascular invasion using the currently available antibodies [27]. This study highlights the need for criteria in evaluation of lymphovascular invasion [27]. Thus, a reported incidence of lymphovascuar invasion for colonic carcinomas was quite variable from $0 \%$ to $31 \%$ using $\mathrm{H}$. and $\mathrm{E}$. sections and immunostained sections for D2-40 and CD 31 [27]-[29]. For a better immunocytochemical study, better antibodies with a higher specificity and sensibility than the currently available sources are warranted. Nevertheless, lymphovascular invasion in colonic carcinoma still remains as a strong stage-independent prognostic marker [30] [31] and this immunocytochemical study has to be pursued in the future.

\section{Acknowledgements}

I want to express my sincere thanks to Dr. Ov Slayden, Division of Reproductive Sciences, Oregon National Primate Center, Beaverton, OR for allowing me to use his laboratory to perform immunocytochemical staining. This study was supported in part by ONPRC Core Grant: NIH RR 000163.

\section{References}

[1] Rosai, J. (2004) Large Bowel, Carcinoma. In: Rosai, J., Ed., Rosai and Ackerman’s Surgical Pathology, 9th Edition, Mosby, St. Louis, 810-825.

[2] Shida, H., Ban, H, Matsumoto, M., Masuda, K., et al. (1992) Prognostic Significance of Location of Lymph Node Metastasis in Colorectal Cancer. Diseases of Colon and Rectum, 32, 1046-1050. http://dx.doi.org/10.1007/BF02252994

[3] Morodomi, T., Isomoto, H., Shirouzu, K., Kageyama K., et al. (1989) An Index for Estimating the Probability of Lymph Node Metastasis in Rectal Carcinoma. Cancer, 63, 539-543. 
http://dx.doi.org/10.1002/1097-0142(19890201)63:3<539::AID-CNCR2820630323>3.0.CO;2-S

[4] Futamura, M., Takagi, Y., Koumura, H., Kida H., et al. (1998) Spread of Colorectal Cancer Micrometastasis in Regional Lymph Node by Reverse Transcriptase-Polymerase Chain Reaction for Carcinoembryonic Antigen and Cytokeratin 20. Journal of Surgical Oncology, 68, 34-40.

http://dx.doi.org/10.1002/(SICI)1096-9098(199805)68:1<34::AID-JSO8>3.0.CO;2-M

[5] Andreola, S., Leo, E., Belli, F., Gallino, G., et al. (2001) Adenocarcinoma of the Lower Third of the Rectum: Metastasis in Preliminary Result on Early Tumor Recurrence. Annul of Surgical Oncology, 8, 413-417. http://dx.doi.org/10.1007/s10434-001-0413-X

[6] Wang, H.S., Liang, W.Y., Lin, T.C., et al. (2005) Curative Resection of $\mathrm{T}_{1}$ Colorectal Carcinoma: Risk of Lymph Node Metastasis and Long-Term Prognosis. Diseases of the Colon and Rectum, 48, 1182-1192.

http://dx.doi.org/10.1007/s10434-001-0413-X

[7] Ishii, M., Ota, M., Saito, S., Kinugasa, Y., et al. (2009) Lymphatic Vessel Invasion Detected by Monoclonal Antibody D2-40 as a Predictor of Lymph Node Metastasis in $\mathrm{T}_{1}$ Colorectal Cancer. International Journal of Colorectal Disease, 24, 1069-1074. http://dx.doi.org/10.1007/s00384-009-0699-x

[8] Imada, K., Shimokawa, K., Ikeda, T., Hayashi, M., et al. (1991) Development of Liver Metastasis in Colorectal Carcinoma. With Special Reference to Venous Invasion and Basement Membrane Laminin. Acta Pathologica Japonica, 41, 2240-2245.

[9] Fong, Y. and Blumgart, L.H. (1998) Hepatic Colorectal Metastasis: Current Status of Surgical Therapy. Oncology, 12, 1489-1498.

[10] Stacker, S.A., Achen, M.G., Jussila, L., Baldwin, M.E. and Alitalo, K. (2002) Lymphangiogenesis and Cancer Metastasis. Nature Rev Cancer, 2, 573-583. http://dx.doi.org/10.1038/nrc863

[11] He, Y., Rajantie, I., Ilmonen, M., Makinen, T., et al. (2004) Preexisting Lymphatic Endothelium but Not Endothelial Progenitor Cells Are Essential for Tumor Lymphangiogegnesis and Lymphatic Metastasis. Cancer Research, 64, 3737-3740. http://dx.doi.org/10.1158/0008-5472.CAN-04-0088

[12] Sharinen, P., Tammela, T., Karkkanen, M.J. and Alitalo, K. (2004) Lymphatic Vasculature: Development, Molecular Regulation and Role in Tumor Metastasis and Inflammation. Trends in Immunology, 25, 387-395. http://dx.doi.org/10.1016/j.it.2004.05.003

[13] Tomita, T. (2008) Immunocytochemical Localization of Lymphatic and Venous Vessels in Colonic Polyps and Adenomas. Digestive Diseases and Science, 53, 1880-1885. http://dx.doi.org/10.1007/s10620-007-0078-9

[14] Quirke, P. and Morris, E. (2007) Reporting Colorectal Carcinoma. Histopathology, 50, 103-112. http://dx.doi.org/10.1111/j.1365-2559.2006.02543.x

[15] Zimmerman, R.L., Ross, H.M. and Gausa, R.E. (2004) Identification of Lymphatic Vessels in Malignant, Adenomatous and Normal Colonic Mucosa Using the Novel Immunostain D2-40. Oncology Report, 11, 47-50.

[16] Zanetta, L., Marcus, S.G., Vasile, J., Dobryansky, M., Cohen, H., et al. (2000) Expression of von Willebrand Factor, an Endothelial Cell Marker, Is Up-Regulated by Angiogenesis Factors: A Potential Method for Objective Assessment of Tumor Angiogenesis. International Journal of Cancer, 85, 281-288.

http://dx.doi.org/10.1002/(SICI)1097-0215(20000115)85:2\%3C281::AID-IJC21\%3E3.0.CO;2-3

[17] Fiedler, U., Christian, S., Koidl, S., Kerjaschki, D. and Emmett, M.S. (2006) The Sialomucin CD 34 Is a Marker of Lymphatic Endothelial Cells in Human Tumors. American Journal of Pathology, 168, 1045-1053. http://dx.doi.org/10.2353/ajpath.2006.050554

[18] Matsumoto, K., Nakayama, Y., Inoue, Y., Minagawa, N., et al. (2007) Lymphatic Microvessels Density Is an Independent Prognostic Factor in Colorectal Carcinoma. Diseases of the Colon and Rectum, 50, 308-314. http://dx.doi.org/10.1007/s10350-006-0792-y

[19] Kaneko, I., Tanaka, S., Oka, S., Kawamura, T., et al. (2007) Lymphatic Vessels Density at the Site of Deepest Penetration as a Predictor of Lymph Node Metastasis in Submucosal Colorectal Carcinoma. Diseases of the Colon and Rectum, 50, 13-21. http://dx.doi.org/10.1007/s10350-006-0745-5

[20] Liang, P., Hong, J.W., Ubukata, H., Liu, H.R., et al. (2006) Increased Density and Diameter of Lymphatic Vessels Correlate with Lymph Node Metastasis in Early Stage Invasive Colorectal Carcinoma. Virchows Archives, 448, 570573. http://dx.doi.org/10.1007/s00428-006-0166-9

[21] Gao, Y., Zhang, W.X., Mu, D.B., Yuan, Y.P., et al. (2008) Distribution of Angiogenesis and Lymphangiogenesis in Gastrointestinal Intramucosal Tumors. Annuls of Surgical Oncology, 15, 1117-1123. http://dx.doi.org/10.1245/s10434-007-9752-6

[22] Longgatto-Filho, A., Pinheiro, C., Fferreira, L., Scapulatempo, C., et al. (2008) Peritumoral, but Not Intratumoral Lymphatic Vessel Density and Invasion Correlate with Colorectal Carcinoma Poor-Outcome Markers. Virchows Archives, 452, 133-138. http://dx.doi.org/10.1007/s00428-007-0550-0 
[23] Minsky, B.D., Miles, C., Rich, T.A. and Ticht, A. (2008) Lymphatic Invasion Is an Independent Prognostic Factor for Survival Incolorectal Carcinoma. International Journal of Radiological Oncology and Biological Physics, 17, 311-318. http://dx.doi.org/10.1016/0360-3016(89)90445-8

[24] Kenny, B.C. and Jain, D. (2008) Identification of Lymphatics in within the Colonic Lamina Propria in Inflammation and Neoplasia Using the Monoclonal Antibody D2-40. Yale Journal of Biology and Medicine, 81, 103-113.

[25] Smith, K., Jones, P., Burke, D.A. Treanor, D. and Finan, P.J. (2011) Lymphatic Vessel Distribution in the Mucosa and Submucosa and Potential Implication for $\mathrm{T}_{1}$ Colorectal Tumors. Diseases of Colon and Rectum, 54, 35-40. http://dx.doi.org/10.1007/DCR.0b013e3181fb0e7a

[26] Duff, S.E., Jeziorska, M., Jumar, S., Haboubi, N., et al. (2006) Lymphatic Vessel Density, Microvessel Density and Lymphagiogenic Growth Factor Expression in Colorectal Cancer. Colorectal Disease, 9, 793-800. http://dx.doi.org/10.1111/j.1463-1318.2006.01199.x

[27] Harris, E.I., Lewin, D.N., Wang, H.L., Lauwers, G.Y., Srivastava, A., Shyr, Y., et al. (2008) Lymphovascular Invasion in Colorectal Cancer: An Interobserver Variability Study. American Journal of Surgical Pathology, 32, 1816-1821. http://dx.doi.org/10.1111/j.1463-1318.2006.01199.x

[28] Messenger, D.E., Driman, D.K., McLeod, R.S., Riddel, R.H. and Kirsch, R. (2011) Current Practice Patterns among Pathologists in the Assessment of Venous Invasion in Colorectal Cancer. Journal of Clinical Pathology, 64, 983-989. http://dx.doi.org/10.1136/jclinpath-2011-200156

[29] Betge, J., Pollheimer, M.J., Lindtner, R.A., Kornprat, P., Schlemmer. A., et al. (2012) Intramural and Extramural Vascular Invasion in Colorectal Cancer. Cancer, 43, 628-638. http://dx.doi.org/10.1002/cncr.26310

[30] Losi, L., Ponti, G., Gregoria, C.D., Marino, M., Rossi, G., et al. (2006) Prognostic Significance of Histological Fea-

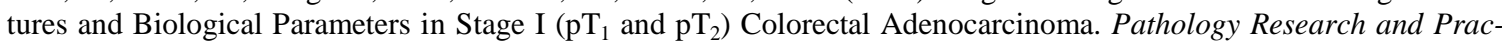
tice, 202, 663-670. http://dx.doi.org/10.1016/j.prp.2006.05.003

[31] Meguerditchain, A.N., Bairati, I., Lagace, R., Harel, F., Dahl, O., et al. (2005) Prognostic Significance of Lymphovascular Invasion in Surgically Cured Rectal Carcinoma. American Journal of Surgery, 189, 707-713. http://dx.doi.org/10.1016/j.amjsurg.2005.03.012 
Scientific Research Publishing (SCIRP) is one of the largest Open Access journal publishers. It is currently publishing more than 200 open access, online, peer-reviewed journals covering a wide range of academic disciplines. SCIRP serves the worldwide academic communities and contributes to the progress and application of science with its publication.

Other selected journals from SCIRP are listed as below. Submit your manuscript to us via either submit@scirp.org or Online Submission Portal.
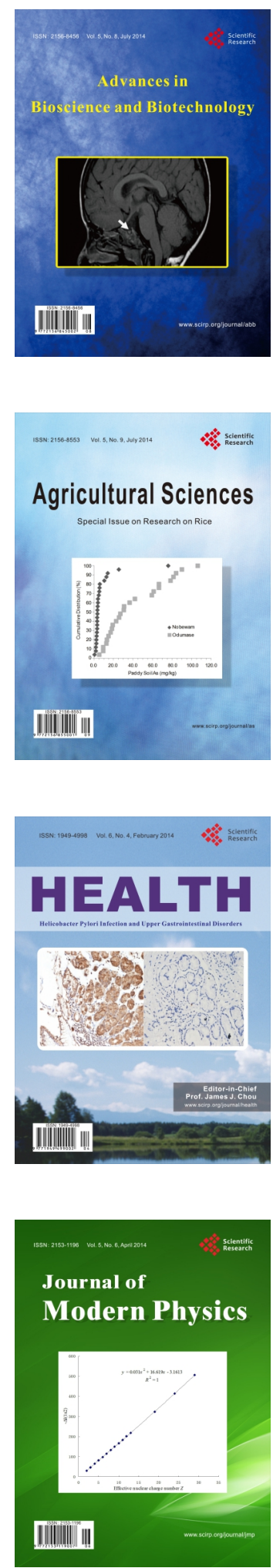
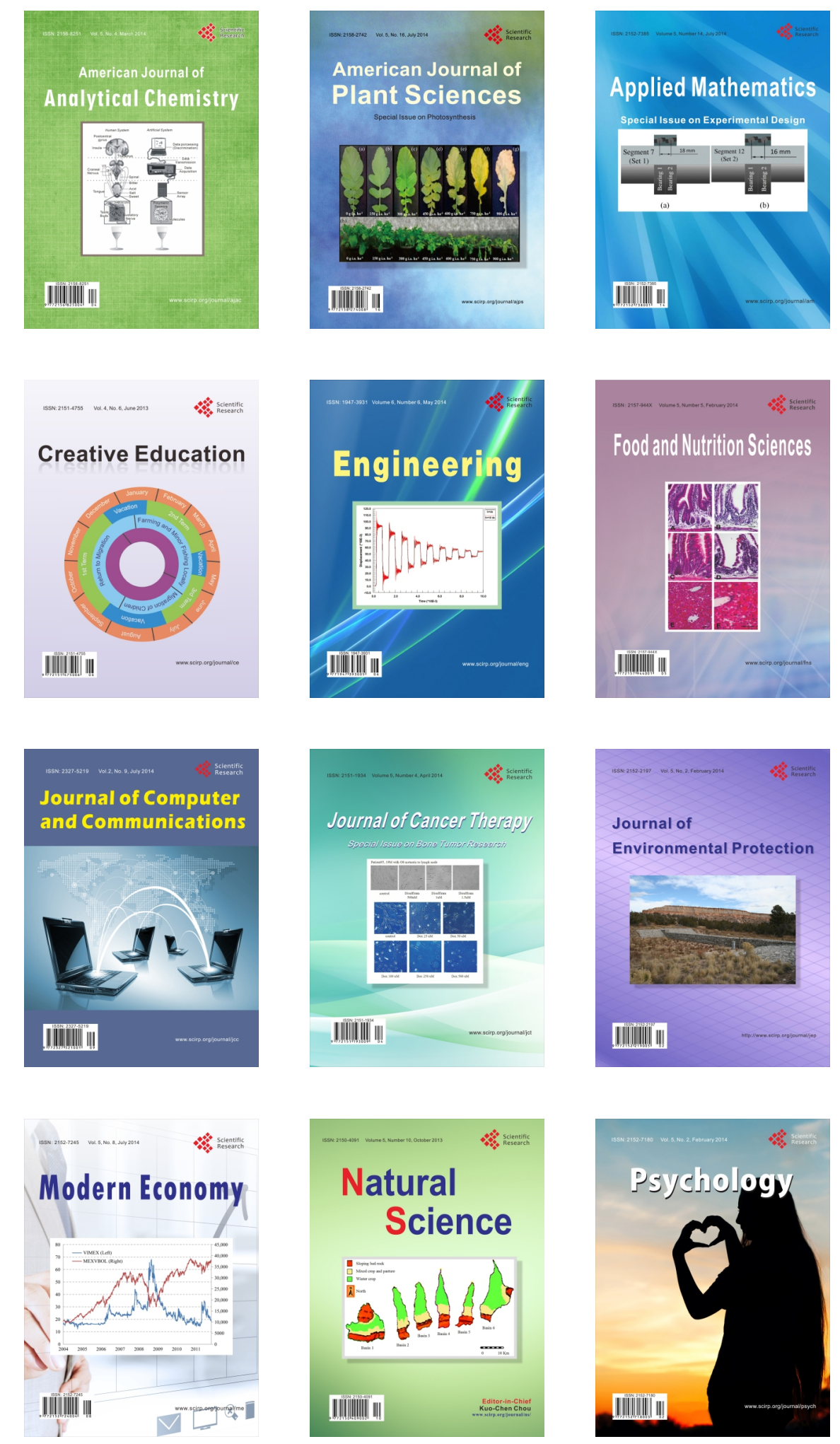